\title{
Schema Redescription in Cellular Automata: Revisiting Emergence in Complex Systems
}

\author{
Manuel Marques-Pita \\ School of Informatics and Computing \\ Indiana University (USA) and \\ Instituto Gulbenkian de Ciência (Portugal) \\ Email: marquesm@indiana.edu
}

\author{
Luis M. Rocha \\ School of Informatics and Computing \\ Indiana University (USA) and \\ Instituto Gulbenkian de Ciência (Portugal) \\ Email: rocha@indiana.edu
}

\begin{abstract}
We present a method to eliminate redundancy in the transition tables of Boolean automata: schema redescription with two symbols. One symbol is used to capture redundancy of individual input variables, and another to capture permutability in sets of input variables: fully characterizing the canalization present in Boolean functions. Two-symbol schemata explain aspects of the behaviour of automata networks that the characterization of their emergent patterns does not capture. We use our method to compare two well-known cellular automata rules for the density classification task [1]: GKL [2], [3] and GP [4]. We show that despite having very different emergent behaviour, these rules are very similar. Indeed, GKL is a special case of GP. Therefore, we demonstrate that it is more feasible to compare cellular automata via schema redescriptions of their rules, than by looking at their emergent behaviour, leading us to question the tendency in complexity research to pay much more attention to emergent patterns than to local (micro-level) interactions.
\end{abstract}

\section{INTRODUCTION}

The intersection of biology and computer science has been fertile for some time. Indeed, von Neumann was a member of the mid-twentieth century Cybernetics group [5], whose main focus was the understanding of natural and artificial systems in terms of communication and control. Most early computer science developments were inspired by the models of cognition that orbited this group [6]. Since then, the need to understand how biological systems are able to control and transmit information throughout the huge number of components that comprise them has only increased. The study of complex network dynamics has received much attention in the last two decades. From pioneering work on networks of automata [7]-[9] to recent models of genetic regulation [10][13], it is clear that to understand and control the biological organization, it is useful to study models based on complex networks of automata [14]-[16].

There has been much progress in understanding the structure of natural networks - at the level of their topology [17], [18] or of their more fine-grained motifs [19] - as well as on modelling biological systems as networks of automata. Yet, we are still to fully grasp how complex networks "compute" and how to harness them to perform specific tasks [20]. The need for a better understanding of collective computation in complex networks has been identified in many areas. For instance, the way plants adjust stomatal apertures for efficient gas exchanges on leaf surfaces is statistically indistinguishable from the dynamics of automata that compute [21], and the high degree of connectivity in biochemical intracellular signal transduction networks endows them with the capability of emergent nontrivial classification-via collective computation [22]. However, not much work in automata models of complex networks has focused on how interactions at the local level of components are linked to collective behaviour.

We show that eliminating redundancy in the transition tables of local automata reveals the loci of control of collective dynamics, better than via the characterization of collective behaviour, emergent patterns, and the like. Our mechanism to eliminate redundancy is schema redescription [23], [24], which we expand here with an additional symbol as explained below. Schemata remove redundancy from automata transition tables, leaving only input states, or groups of input states that control state-transitions, known as their canalizing inputs [25]. Moreover, we discuss how schema redescription can reveal the links between local and collective behaviour in automata networks (here cellular automata). We exemplify our approach with the classical problem of collective computation in cellular automata: the density classification task [1].

\section{Cellular Automata}

A $\kappa$-state automaton is an integer variable $x \in\{0, \ldots, \kappa-1\}$, whose value (or state) is computed at time $t+1$ via a statedetermined transition function $F$, which takes as input the state of a set of $n$ automata at time $t$. When $x=0$ we refer to it as being in the quiescent state; any other state is regarded as an active state. To study the dynamics of systems of many simple elements (known as complex systems), it is common to study networks of interconnected automata. One way to do this is to arrange automata in a regular lattice of $N$ cells, which we refer to as cellular automata, a formalization of a complex dynamical system with its origins in the work of Ulam and von Neumann [26]. In a homogeneous cellular automaton (CA), which we use here, each cell is defined by the same $\kappa$-state automaton and is connected to $n$ neighbour cells in the lattice (including itself). Therefore, there are $\kappa^{n}$ possible local neighbourhood configurations (LNCs). The transition function for every cell, $F=\left\{f_{j}\right\}$, can thus be defined as a look-up table (LUT) where each entry, $f_{j}, j \in\left\{1, \ldots, \kappa^{n}\right\}$, is an assignment of a possible input LNC, or condition part, to an output state 
$x_{i}(t+1) \in\{0, \ldots, \kappa-1\}$. In binary CAs $(\kappa=2)$ it is possible to classify individual cell state transitions in three categories: (1) preservations, where a cell does not change its state in the next time step, i.e. $x_{i}(t)=x_{i}(t+1)$; (2) generations, in which the cell goes from the quiescent to the active state; and (3) annihilations, where the cell goes from the active to the quiescent state. The initial configuration (IC) of states of a CA lattice is typically random. In the CAs we consider here, cells in the lattice update their states synchronously.

\section{SCHEMA REDESCRIPTION}

We previously proposed schema redescription with one symbol as a method to relate an automaton's LUT to the collective dynamics it produces in one- and two-dimensional CA lattices [23], [24]. Here we introduce two-symbol schemata. The basic idea is simple: LUTs are compressed into parsimonious schemata with two extra symbols that capture redundant input states. One symbol captures the irrelevance of some inputs in some situations; the other the permutability of subsets of inputs. Only the input states that effectively determine the automaton's state transitions remain. A related notion of canalizing inputs exists in the context of modelling biochemical networks, e.g. those that regulate the expression patterns of genes. Indeed, automata with canalizing inputs have been shown to stabilize the dynamics of Boolean models of genetic regulation [13], [25], [27].

\section{A. Wildcard Schemata}

To unpack the notion of schema, consider a Boolean automaton $x$, whose state-transition function depends on the states of six Boolean inputs (possibly other automata). Figure 1 A depicts the subset of LUT entries $\left(f_{\alpha}\right)$ for automaton $x$, that lead to state-transition $x(t+1)=1$.

We first compress LUT entries using schemata that are like LUT entries, but allow an additional wildcard (\#) symbol (represented graphically in grey) to appear in their condition part. A wildcard input means that any valid state is accepted for it, with no effect on the state-transition. This results in a redescription of the set of LUT entries, $F$, for an automaton $x$ of $n$ inputs into a smaller set of wildcard schemata, $F^{\prime} \equiv\left\{f_{v}^{\prime}\right\}$ (see Figure $1 \mathrm{~B}$ ). The set $F^{\prime}$, for a given automaton also contains the original entries in its LUT, $F$, that could not be redescribed by wildcard schema. Each specific wildcard schema $f_{v}^{\prime}$ redescribes a subset of entries in the original LUT, denoted by $\Upsilon_{v} \equiv\left\{f_{\alpha}: f_{\alpha} \longmapsto f_{v}^{\prime}\right\}$ ( $\longmapsto$ means "is redescribed by"). Wildcard schemata are minimal in the sense that none of the (non-wildcard) inputs in the condition part of a schema can be "raised" to the wildcard status and still ensure the automaton's transition to the same state. Because wildcard schemata are minimal, it follows that for any two wildcardschemata $f_{v}^{\prime}$ and $f_{\phi}^{\prime}, \Upsilon_{v} \nsubseteq \Upsilon_{\phi} \wedge \Upsilon_{\phi} \nsubseteq \Upsilon_{v}$. Because wildcard schemata are minimal and unique, they are equivalent to the set of prime implicants obtained during the first step of the Quine \& McCluskey Boolean minimization algorithm [29]. In other words, a schema is unique in the sense that the subset of LUT entries it redescribes is not fully redescribed by any other schema. However, in general $\Upsilon_{v} \cap \Upsilon_{\phi} \neq \emptyset$. This means that schemata can overlap in terms of the LUT entries they describe. In Figure 1. $\Upsilon_{1} \equiv\left\{f_{1}, f_{5}, f_{9}, f_{13}\right\}$ and $\Upsilon_{9} \equiv\left\{f_{4}, f_{5}, f_{6}, f_{7}\right\}$, therefore $\Upsilon_{1} \cap \Upsilon_{9} \equiv\left\{f_{5}\right\}$.

Our redescription methodology derives from the work of John Holland on condition/action rules to model inductive reasoning in cognitive systems [28]. However, the same idea had been developed previously for the minimization of circuits in electrical engineering [29]. It was also used by Valiant [30] when introducing Probably Approximately Correct (PAC) learning.
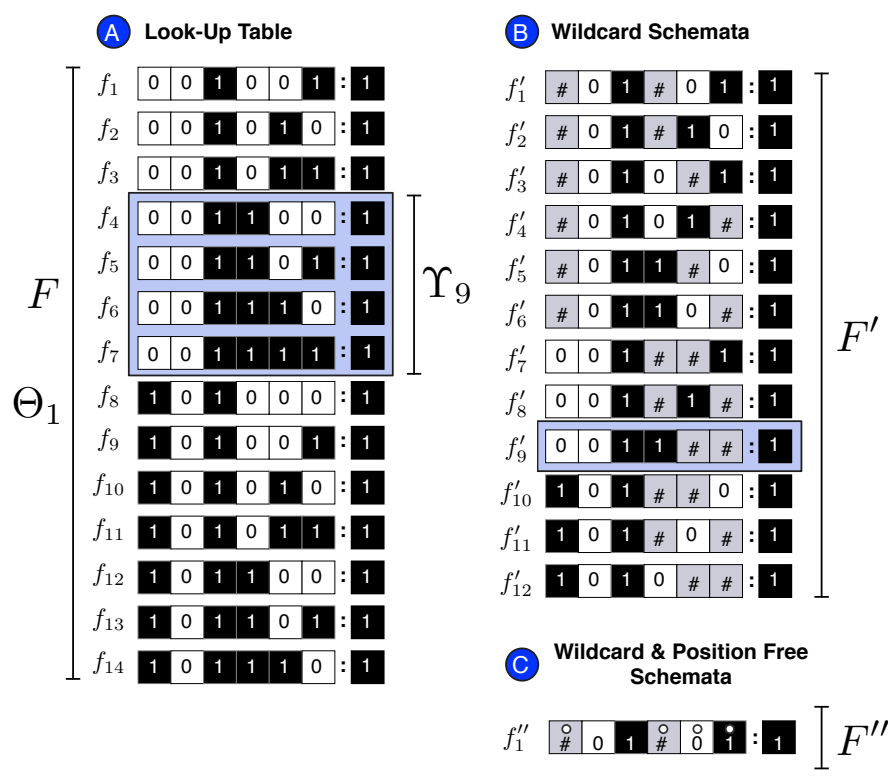

Fig. 1. A set of LUT entries with transition to 1, redescribed. White (black) states are 0 (1). Wildcards are grey. Notice that $\Upsilon_{9} \equiv\left\{f_{4}, f_{5}, f_{6}, f_{7}\right\}$. Using the additional position-free symbol, the entire set $F^{\prime}$ is compressed into a single two-symbol schema: $f_{1}^{\prime \prime}$. Any permutation of the inputs with the position-free symbol results in a schema in $F^{\prime}$. Since there is only one set of marked inputs, the position-free symbol does not require an index.

A wildcard schema can always be expressed as a logical conjunction of literals (logical variables or their negation). Since such a schema is a prime implicant, it follows that all of its literals are essential to determine the automaton's state transition. We refer to the literals (input states) in a wildcard schema as its essential input states or enputs for short. For instance, schema $f_{9}^{\prime}$ of the example of figure 1 has 4 enputs: the first 4 cells in its condition part. The set $F^{\prime}$ can be expressed as a logical disjunctive normal form (DNF) - that is, a disjunction of conjunctions.

\section{B. Two-Symbol Schemata}

Here we introduce a further redescription of $F^{\prime}$ that results in a set of two-symbol schemata $F^{\prime \prime} \equiv\left\{f_{\theta}^{\prime \prime}\right\}$ (Figure 1 $\mathrm{C}$ ). The position-free symbol $\left(\mathrm{O}_{m}\right)$ above inputs in a condition part means that any pair of inputs thus marked can "switch places" without affecting the automaton's state-transition. The index of the position-free symbol, when necessary, is used to differentiate among distinct subsets of inputs whose 
elements can only switch places with each other. A twosymbol schema $f_{\theta}^{\prime \prime}$ redescribes a set $\Theta_{\theta} \equiv\left\{f_{\alpha}: f_{\alpha} \longmapsto f_{\theta}^{\prime \prime}\right\}$ of LUT entries of $x$. In the same way a wildcard schema captures a subset $\Upsilon_{v}$ of LUT entries, a two-symbol schema redescribes a subset of wildcard schemata 1 Therefore $f_{\theta}^{\prime \prime}$ captures the degree of symmetry or group invariance in the set of wildcard schemata contained in $\Theta_{\theta}^{\prime}$ [31]. We refer to the subsets of such marked inputs in a two-symbol schema as group-invariant enputs, which may include wildcard symbols marked with the position-free symbol. Since each two-symbol schema compresses a number of wildcard schemata, it follows that for a given automaton $x,\left|F^{\prime \prime}\right| \leq\left|F^{\prime}\right| \leq|F|=2^{k}$.

After redescription, all redundancy is removed in the form of the two symbols. In a given schema, a wildcard input alone is not essential by definition: it is never an enput, while an individual input set up to a Boolean state always is. However, when a wildcard input is marked with a position-free symbol, then it is part of a group-invariant enput defined by the set of inputs thus marked. In this case, the enput identifies a group invariance property, such as "as long as two of these inputs are false". To summarize, the set of wildcard schemata $F^{\prime}$ of an automaton $x$ captures its enputs, while the addition of the position-free symbol captures group invariant relationships in subsets of wildcard schemata. In our working example, the resulting two-symbol schema (Figure 11) reveals that the automaton's transition to true is determined only by a subset of its six inputs: as long as inputs 2 and 3 are false and true, respectively, and among the others at least one is true and another is false, the automaton will transition to true. Note that such minimal input constraints are not obvious by looking at the LUT of the example automaton.

In previous work, we used wildcard schemata to identify conceptual properties of known solutions to a well-studied problem that requires non-trivial collective computation: the density classification task in homogeneous cellular automata. By uncovering these properties, we were able to devise a genetic algorithm that operated on a search space of wildcard schemata, rather than the space of LUTs. We found that the space of schemata is much more amenable to search, with very good correlations among good solutions to the problem. This way, we found some of the best solutions to this problem [23], [24]. Our aims here are: (1) introduce twosymbol schemata; (2) demonstrate that they are more useful to explain and control the function and behaviour of CAs than existing techniques to analyse collective behaviour, and (3) it is more feasible to compare automata transition functions via their schemata redescriptions, than by looking at their emergent or collective behaviour.

\footnotetext{
${ }^{1}$ Given a two-symbol schema, $f_{\theta}^{\prime \prime}$, the wildcard schemata it redescribes can be denoted by the set $\Theta_{\theta}^{\prime}$. It is useful to look at set $\Theta_{\theta}^{\prime}$ as a matrix where rows correspond to wildcard schemata, and columns to their inputs. The last column is the state transition of $f_{\theta}^{\prime \prime}$, and may therefore be omitted. The wildcard-schemata rows of matrix $\Theta_{\theta}^{\prime}$ have the same number of zeroes, ones and wildcards. For any pair of inputs $(i, j)$ in $f_{\theta}^{\prime \prime}$ that are marked with the same position-free symbol, the corresponding $i^{\text {th }}$ and $j^{\text {th }}$ columns of $\Theta_{\theta}^{\prime}$ can be switched, and the rows of the resulting matrix reordered to obtain $\Theta_{\theta}^{\prime}$ again.
}

\section{The Density Classification Task (DCT)}

The density classification task (DCT) is the most studied example of collective computation in CAs. The goal is to find a binary CA that can classify the majority state in a random IC. If the majority of cells in the IC is in the quiescent (active) state, after a number of time steps the lattice should converge to a homogeneous state where every cell is in the quiescent (active) state. Since the outcome could be undecidable in lattices with even number of cells $(N)$, lattices with an odd number of cells are used instead. Devising CA rules that perform this task is not trivial, because cells in a CA lattice update their states based on local neighbourhood information, the states of 7 input Boolean variables in this case. Therefore, this task requires information to be transferred across time and (lattice) space to achieve a correct global classification. The performance of CA rule $F$, on $C$ initial configurations of length $N$ is denoted by $\mathcal{P}_{N}^{C}(F)$. The definition of the DCT used in our studies comes from [32].

\section{EXPLANATION IN COMPLEX SySTEMS}

The complex systems community has produced methodologies to characterize space-time dynamics in CAs to study emergent behaviour, often using the DCT as a test-case [20]. The most well-known such methodology is the computational mechanics framework (CM) [33], [34], used to extract regular domains: repeating patterns in the space-time dynamics, formally defined as sets of regular languages. It has been argued that in order to explain emergent behaviour, we should look at the boundaries between domains, known as particles, as this is where information is exchanged in the CA lattice [35]. Therefore, collective behaviour in CA dynamics is characterized by finite catalogues of particles and their interaction rules, which define the emergent computation implemented by a given CA [20], and can be seen to possess some features of linguistic representation, though not in fully symbolic forms [36]. Figure 2 depicts the catalogue of particles for the $F_{\mathrm{GKL}}$ rule [2], [3], which we describe below.

Other methods exist to detect regularities in CA spacetime dynamics, typically statistical and information theoretical filters that uncover patterns that correspond to the particles found via CM [37], [38]. But when it comes to understanding and controlling collective behaviour, a key issue is concerned with finding the best explanatory device to use. Do we focus exclusively on the emergent patterns of collective dynamics (the "emergentist" paradigm), as CM and related methodologies do? Or can we gain explanatory advantages by paying more attention to the lower level of local transition functions?

In the remaining of this article we pursue the second approach, towards a hierarchical methodology that seeks to link local interactions to global behaviour, ultimately allowing us to better understand and control the collective dynamics of complex systems modelled as automata networks. 


\begin{tabular}{|c|c|c|c|c|}
\hline \multicolumn{3}{|c|}{ Regular Domains } & \multicolumn{2}{|c|}{ Particle Interactions } \\
\hline$\Lambda^{0}=0^{*}$ & $\Lambda^{1}=1^{*}$ & * $\Lambda^{2}=(01)^{*}$ & decay & $\mu \rightarrow \delta+\beta$ \\
\hline \multicolumn{3}{|c|}{ Particles (Velocities) } & \multirow{2}{*}{ react } & \multirow{2}{*}{$\begin{array}{l}\alpha+\delta \rightarrow \gamma \\
\beta+\alpha \rightarrow \eta \\
\gamma+\eta \rightarrow \alpha\end{array}$} \\
\hline \multicolumn{2}{|c|}{$\alpha \sim \Lambda^{0} \Lambda^{1}(0)$} & $\beta \sim \Lambda^{2} \Lambda^{0}(1)$ & & \\
\hline \multicolumn{2}{|c|}{$\gamma \sim \Lambda^{0} \Lambda^{2}(3) \quad \bigcirc$} & $\delta \sim \Lambda^{1} \Lambda^{2}(-1) \bigcirc$ & \multirow{2}{*}{$\begin{array}{c}\text { particle } \\
\text { annihilation }\end{array}$} & \multirow{2}{*}{$\begin{array}{l}\gamma+\beta \rightarrow \varnothing_{1} \\
\delta+\eta \rightarrow \varnothing_{2}\end{array}$} \\
\hline \multicolumn{2}{|c|}{$\eta \sim \Lambda^{2} \Lambda^{1}(-3) \bigcirc$} & $\mu \sim \Lambda^{1} \Lambda^{0}(0) \quad \bigcirc$ & & \\
\hline
\end{tabular}

Fig. 2. Catalogue of regular domains, particles (domain boundaries), partic velocities (in parentheses), and particle interactions seen in $F_{\mathrm{GKL}}$ 's spac time behaviour. The colouring assigned to each particle is then used in Figu 3 The notation $p \sim \Lambda^{x} \Lambda^{y}$ means that $p$ is the particle forming the bounda between regular domains $\Lambda^{x}$ and $\Lambda^{y}$. (Adapted from [1].)

\section{Comparing Collective Dynamics}

Most of the known one-dimensional CA rules for the DC were analysed using wildcard schemata in [39]. We four that most of these CAs are process symmetric: they classit equally well ICs with majority 0 s and with majority $1 \mathrm{~s}$. I also found that two of the most well-known high-performir CA rules for the DCT, while observing very distinct collectis behaviour, are nonetheless similar at the local LUT level [39 More specifically, we refer to the human-derived CA $F_{\mathrm{GKL}}[2$ [3], and $F_{\mathrm{GP}}$, derived via genetic programming [4]. Figurt 3 and 4 depict the space-time dynamics of $F_{\mathrm{GKL}}$ and $F_{\mathrm{G}}$ respectively. The performance of these rules on the DCT i $\mathcal{P}_{149}^{10^{5}}\left(F_{\mathrm{GKL}}\right) \approx 0.815$, and $F_{\mathrm{GP}} \mathcal{P}_{149}^{10^{5}}\left(F_{\mathrm{GP}}\right) \approx 0.822$. In th remainder of this article, instead of $F_{\mathrm{GKL}}$ we use the ful] equivalent $F_{\mathrm{GKL}^{\prime}}$, which is the mirror rule of $F_{\mathrm{GKL}}$. That $\mathrm{i}$ exactly the same CA rule, but where the particles traverse tr lattice in the opposite direction, with the same velocities ar interactions (see computationally equivalent CAs in [39]).

The collective behaviour of $F_{\mathrm{GKL}^{\prime}}$ and $F_{\mathrm{GP}}$ is quite distinc while $F_{\mathrm{GKL}^{\prime}}$, after a short transient, leads to the appearance c three domains, the dynamics of the $F_{\mathrm{GP}}$ leads to the appearanc of many more (we have identified at least ten [36], see figu] 41. Because $F_{\mathrm{GKL}^{\prime}}$ only has three domains, from the $\mathrm{Cl}$ perspective, its collective dynamics can be characterized ar explained with a catalogue of six particles: the permutatior without repetition of two out of three possible domains, whes order is relevant Indeed, the collective behaviour of $F_{\mathrm{GK}}$ has been well predicted by the CM framework, using on] six particles [40], [41]. In contrast, $F_{\mathrm{GP}}$ produces many mo] particles; even with just ten domains (it has more), there woul. be ninety possible particles and many potential interactions. Interestingly, even though the collective dynamics of the two rules is apparently (via the CM framework) completely different, we found that $F_{\mathrm{GP}}$ can be easily derived from $F_{\mathrm{GKL}}$, via a small sequence of operations on the wildcard schemata representation of the latter.

The wildcard schemata for $F_{\mathrm{GKL}^{\prime}}$ are shown in Figure 5 Note that most schemata are state-preserving: all state-changes in this automaton are described by two wildcard schemata $\left(f_{5}^{\prime}\right.$ and $\left.f_{10}^{\prime}\right)$. Generations (annihilations) occur only when a

$$
{ }^{2} p=d ! /(d-2) ! \text {, where } d \text { is the the number of domains. }
$$
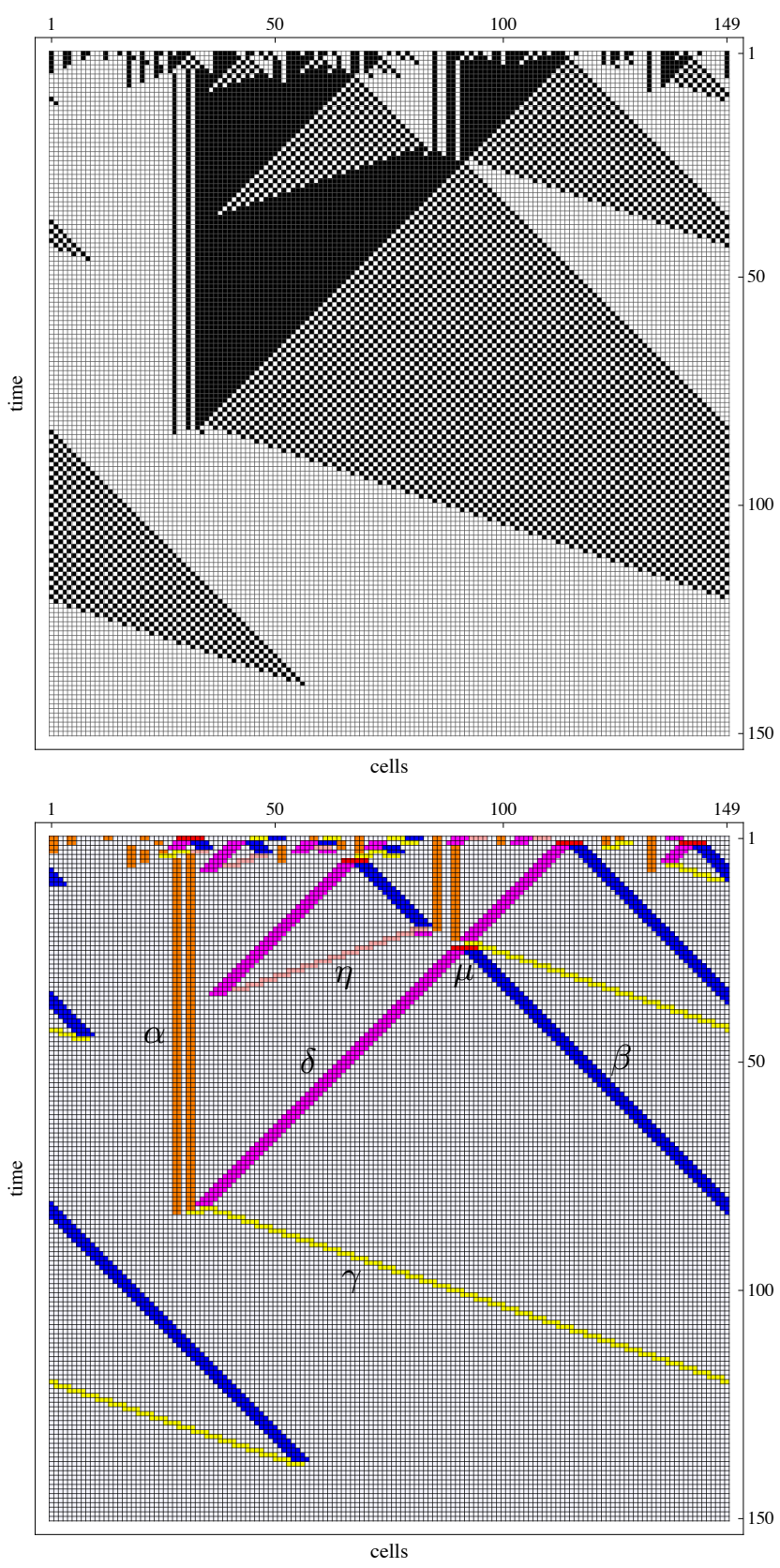

Fig. 3. (Top) A space-time diagram produced by $F_{\mathrm{GKL}}$. (Bottom) The diagram with the regular domains filtered out, leaving only the locations of the particles (coloured using the scheme in Figure 2). Note that particle $\alpha$ (red) lasts for only one time step, after which it decays to particles $\delta$ and $\beta$.

cell in state 0 (1) observes right (left) and right-most (leftmost) neighbours in the opposite state-this is characterized by schemata $f_{5}^{\prime}$ and $f_{10}^{\prime}$. In all other situations the a cell $x_{i}(t+$ 1) $=x_{i}(t)$.

The wildcard schemata for $F_{\mathrm{GP}}$ are shown in Figure 6. In this case, all state changes are characterized by three wildcard schemata: $f_{1}^{\prime}, f_{3}^{\prime}$, and $f_{5}^{\prime}$ for annihilation, and $f_{6}^{\prime}, f_{8}^{\prime}$, and $f_{10}^{\prime}$ for generation. In all other situations the a cell $x_{i}(t+1)=$ $x_{i}(t)$. Using the the two-symbol schemata introduced here, 


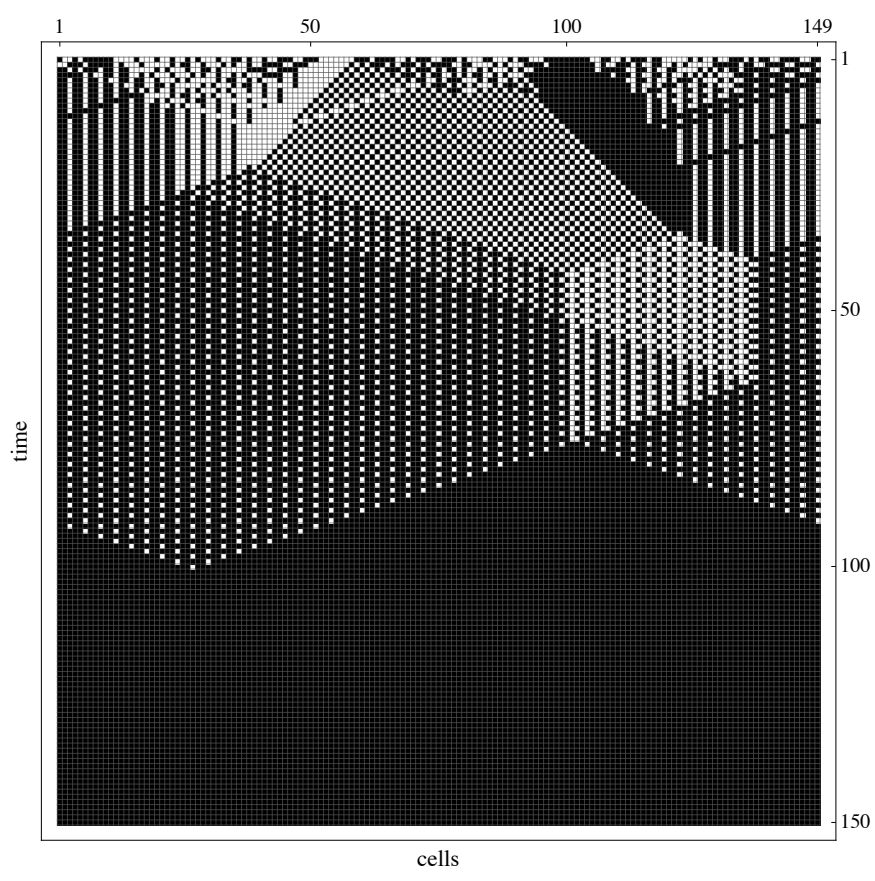

Fig. 4. A space-time diagram produced by $F_{\mathrm{GP}}$

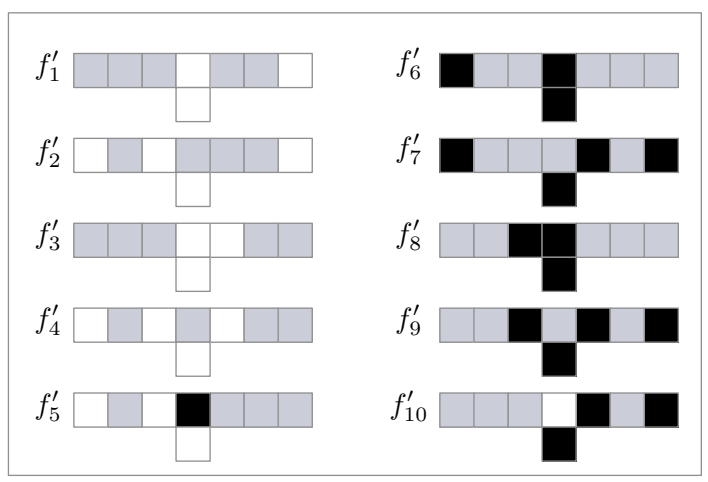

Fig. 5. Wildcard schemata of $F_{\mathrm{GKL}^{\prime}}$. Schemata $f_{5}^{\prime}$ and $f_{10}^{\prime}$ describe all the possible state-changes for a cell; all other situations are preservations. Notice that schemata $f_{2}^{\prime}, f_{4}^{\prime}, f_{7}^{\prime}$ or $f_{9}^{\prime}$, when applicable to a state-change, describe LUT entries already described by $f_{5}^{\prime}$ or $f_{1}^{\prime} 0$, respectively; When prescribing preservations the same schemata are captured with less enputs by $f_{1}^{\prime}, f_{3}, f_{6}^{\prime}$ or $f_{8}^{\prime}$. This means that $f_{2}^{\prime}, f_{4}^{\prime}, f_{7}^{\prime}$ or $f_{9}^{\prime}$ are not essential schemata.

we can appreciate better the similarity between the two rules. Figure 7 depicts the essential state-changing transitions in both rules, which we obtained by via two-symbol schemaredescription, thus removing all redundancy from local dynamics. We can now, for the first time, understand that $F_{\mathrm{GP}}$ is doing something very similar to, and which includes the behaviour of, $F_{\mathrm{GKL}^{\prime}}$ at local level.

Both CA rules can be understood by a single schema for generation and their process-symmetric schema for annihilation. Each such schemata is characterized by two enputs. Moreover, one of the enputs is exactly the same for both CAs: for the generation schema, both CAs depend on the right-most neighbour being 1, and for annihilation schemata, both CAs depend on the left-most neighbour being 0 . The

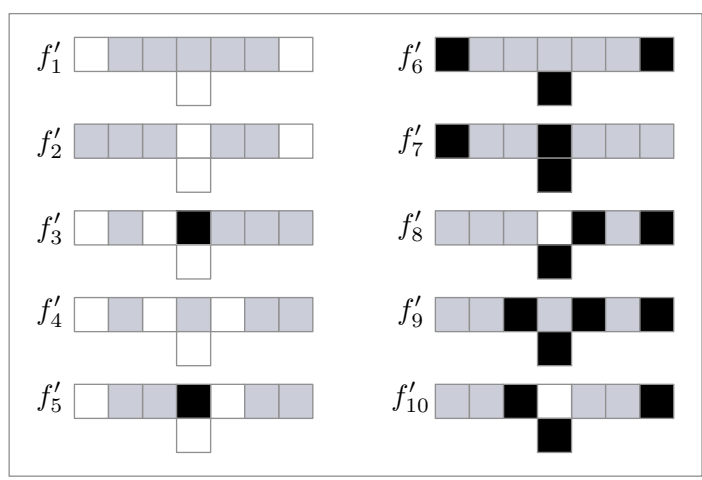

Fig. 6. Wildcard schemata for $F_{\mathrm{GP}} f_{1}^{\prime}, f_{3}^{\prime}, f_{5}^{\prime}, f_{6}^{\prime}, f_{8}^{\prime}$, and $f_{10}^{\prime}$ describe all the possible state-changes for a cell $x_{i}$; all other situations are preservations. Notice that schemata $f_{4}^{\prime}$ and $f_{9}^{\prime}$, when applicable to a state-change, describe LUT entries already described by $f_{3}^{\prime}$ or $f_{5}^{\prime}$ and $f_{8}^{\prime}$ or $f_{10}^{\prime}$, respectively

second (group-invariant) enput in $F_{\mathrm{GP}}$ is also very similar to, and indeed contains the second enput for, $F_{\mathrm{GKL}^{\prime}}$. For the generation schema, $F_{\mathrm{GKL}^{\prime}}$ depends on the immediate-right neighbour being 1 , and for the annihilation schema, $F_{\mathrm{GKL}^{\prime}}$ depends on the immediate-left neighbour being 0 . When it comes to $F_{\mathrm{GP}}$, the second enput is a group-invariant enput: one of three possible input cells in the neighbourhood may be specified (set to 0 or 1). Specifically, For the generation schema, $F_{\mathrm{GP}}$ depends not only on the right-most neighbour being 1, like $F_{\mathrm{GKL}^{\prime}}$, but alternatively, on the immediate-left, immediate-right or left-most neighbours being 1 . Likewise, for the annihilation schema, $F_{\mathrm{GP}}$ depends not only on the left-most neighbour being 0 , like $F_{\mathrm{GKL}^{\prime}}$, alternatively, on the immediateleft, immediate-right or right-most neighbours being 0 .
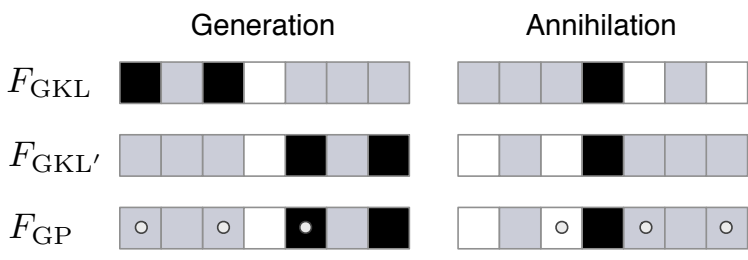

Fig. 7. Two-symbol schemata for $F_{\mathrm{GKL}^{\prime}}\left(F_{\mathrm{GKL}}\right)$ and $F_{\mathrm{GP}}$, characterizing every possible situation where there are state changes for these rules. Note that here we include the state of the updating cell, (even if not an enput in the original schemata) to describe the known state-changing transitions.

\section{Connecting The LeVels}

The similarity between the local-level descriptions of $F_{\mathrm{GKL}^{\prime}}$ and $F_{\mathrm{GP}}$ we discovered, as well as their similar performance leads to a natural question: do the additional regular domains produced by the $F_{\mathrm{GP}}$, play a significant role in its collective computation of the DCT? The following three facts are known: (1) $F_{\mathrm{GP}}$ produces the same three regular domains as $F_{\mathrm{GKL}^{\prime}}$, plus many others; (2) the performance difference between the two rules, while significant, is less than $1 \%$; and (3) the number of possible particles grows very rapidly with number $d$ of domains $(d ! /(d-2) !)$. Therefore, it is reasonable to conclude that many-even perhaps most of the regular domainsproduced by $F_{\mathrm{GP}}$ must be redundant; i.e. the particles they 
produce lead to interactions that have no effect on information transmission across the lattice.

Support for this conclusion comes from different sources. One is simple inspection of the space-time dynamics produced by $F_{\mathrm{GP}}$. Figure 8 depicts a portion of the space-time dynamics for $F_{\mathrm{GP}}$ shown in Figure 4 Notice, for example, the collision between the particle formed by domains $\Lambda^{5}$ and $\Lambda^{3}$ (highlighted in green), and the particle formed by domains $\Lambda^{5}$ and $\Lambda^{6}$ (orange). This collision, in the CM framework perspective, leads to two new particles: one formed by domains $\Lambda^{6}$ and $\Lambda^{4}$, and another formed by domains $\Lambda^{3}$ and $\Lambda^{4}$. However, this collision seems to be redundant, leading to particles that behave exactly as the colliding particles (same velocity, possibly same structure). This is highlighted in Figure 8 by using the same colours for the particles observed before and after the collision. This suggests that the (large) set of possible regular domains in the dynamics of $F_{\mathrm{GP}}$ contains sets of domains that are equivalent, such that collisions among the particles they produce are redundant. In the example above $\Lambda^{5}$ and $\Lambda^{6}$ seem to be equivalent, just like $\Lambda^{3}$ and $\Lambda^{4}$.

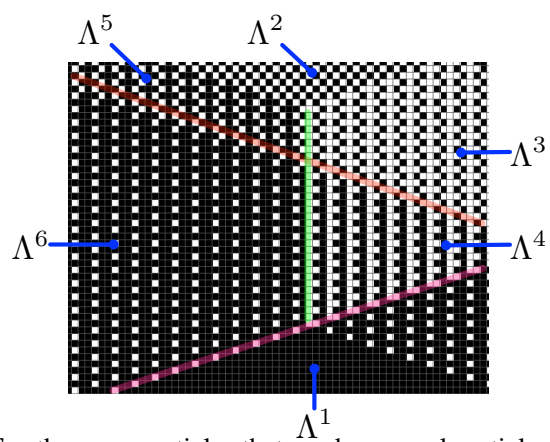

Fig. 8. In $F_{\mathrm{GP}}$ there are particles that produce equal particles after collisions (Highlighted in green (vertical) and orange/purple (inclined)

Our observation is supported further when looking at the schemata for $F_{\mathrm{GP}}$. We can use the knowledge encoded in schemata, to better understand the collective behaviour of the CA. Consider $f_{1}^{\prime}$ in $F_{\mathrm{GP}}$, which enforces that a cell $x_{i}$ in any state whose left- and right-most neighbours are 0 will transition to, or stay 0 at $t+1$ (see Figure 7). This leaves a wide "gap" of five cells in-between the 0 -cells, which can be in any state, without affecting the state-transition of $x_{i}$ to 0 . When this pattern is repeated in the lattice, i.e. every $6^{\text {th }}$ cell is 0 , using the two-symbol schemata obtained instead of the original LUT, we can compute the resulting collective dynamics with incomplete information. Indeed, one key advantage of schema redescription is that it allows the simulation of the dynamics of automata networks from ICs where only the state of some cells is specified, while the state of the others is unknown.

Figure 9 (A) depicts the known dynamics given partial lattice information, where every sixth cell is 0 (except at the lattice borders) and the state of grey cells is unknown. This allows us to know how the initial input pattern will propagate in the CA dynamics. In this case, our lack of knowledge propagates in the lattice, and our partial knowledge of the dynamics eventually disappears completely. In 9 (B) we show that different such patterns can co-exist independently, in the

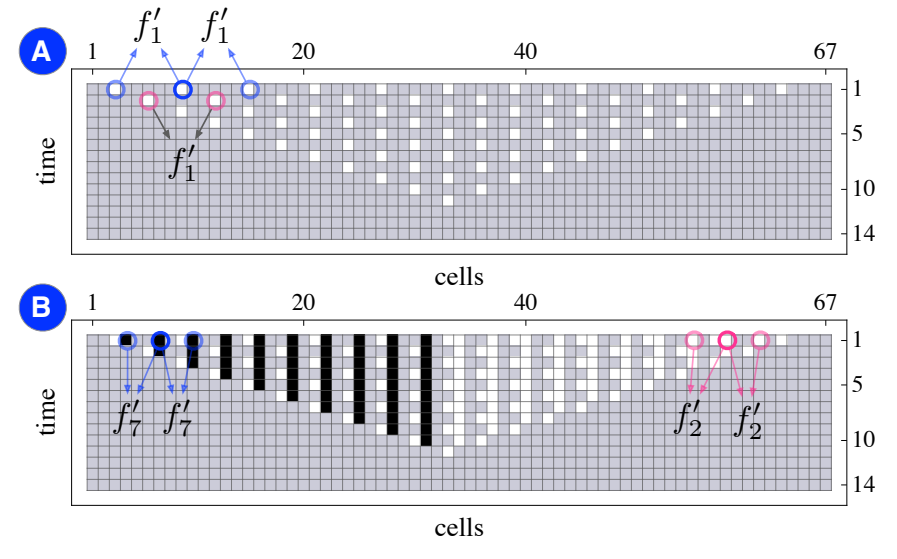

Fig. 9. Dynamically decoupled dynamics of patterns in $F_{\mathrm{GP}}$. See text for description.

previous unknown segments of the lattice. The difference from (A) is that every third cell in the left half of the initial lattice is set to 1 , and the third and every third cell after that is set to 0 , in the right half of the initial lattice (these conditions match schemata $f_{7}^{\prime}$ and $f_{2}^{\prime}$ respectively).

One very interesting observation from computing dynamics with incomplete information, is that many patterns (such as those in Figure 9) are dynamically decoupled in $F_{\mathrm{GP}}$. This means that the space-time patterns produced from schemata $f_{1}^{\prime}, f_{2}^{\prime}$ and $f_{7}^{\prime}$ in $F_{\mathrm{GP}}$ are updated independently: they function as building blocks that can switch places, producing different domains (from the CM perspective) that in reality are formed of some of the same sub-patterns (defined by the same schemata). This is clear in Figure 9 (B) where the patterns from schemata $f_{1}^{\prime}, f_{2}^{\prime}$ and $f_{7}^{\prime}$, coexist independently of one another. Since some of the schemata for $F_{\mathrm{GP}}$ have large "gaps" wherein the states of the cells are irrelevant, if particles collide inside those gaps, some of the decoupled underlying patterns are unaffected, leading to equivalent domains and redundant collisions such as what we apparently observe in Figure 8.

In contrast, rule $F_{\mathrm{GKL}^{\prime}}$ does not easily lead to dynamic decoupled patterns and redundant collisions, since its schemata do not leave large enough "gaps" in the lattice for other pat-

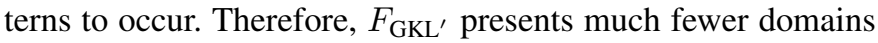
than rule $F_{\mathrm{GP}}$. But since the domains obtained by rule $F_{\mathrm{GP}}$ are the result of schemata with a large amount of canalization leading to large "gaps" that can be in any state, many of the obtained distinct domains are dynamically equivalent.

This observation is also supported by the schema redescription of additional, better-performing rules for the DCT: $F_{\mathrm{MM} 0802}$ [23] and $F_{\mathrm{WO}}$ [42], with performances $\mathcal{P}_{149}^{10^{5}}\left(F_{\mathrm{MM} 0802}\right) \approx 0.845$ and $\mathcal{P}_{149}^{10^{5}}\left(\overline{F_{\mathrm{WO}}}\right) \approx 0.889$. The twosymbol (state-changing) schemata for these rules are depicted in Figure 10 . As we can see, for the DCT, increasingly betterperforming rules become "less canalized". This means that they are very sensitive to noise in ICs (as we would expect for the DCT): bit-flips or incomplete state-specification have a high chance of leading to wrong classification (in DCT terms). Since noise is not used in their performance computation and 
fitness evaluations, these rules find advantages in becoming increasingly specific to the initial conditions they respond differently to. Therefore, they have a higher number of schemata with more enputs and therefor less canalization to describe their behaviour. The dynamics of these CAs do not produce the large numbers of domains as observed in $F_{\mathrm{GP}} \cdot 3$

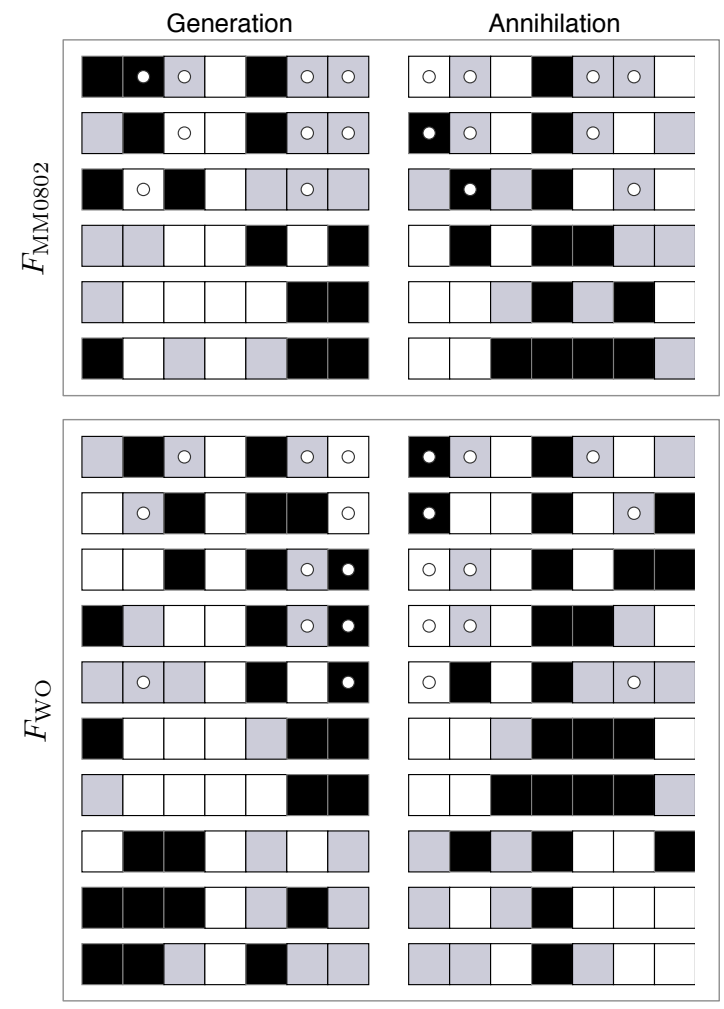

Fig. 10. 2-symbol schemata of $F_{\mathrm{MM} 0802}$ and $F_{\mathrm{WO}}$, characterizing every possible situation where there are state changes for this rule.

\section{DISCUSSION}

Two-symbol schema redescription allowed us for the first time to understand that despite dramatically distinct collective dynamics (emergent behaviour), the $F_{\mathrm{GP}}$ rule is a more general version of the $F_{\mathrm{GKL}^{\prime}}$ rule. The statistically significant, albeit small, performance improvement of $F_{\mathrm{GP}}$ over $F_{\mathrm{GKL}^{\prime}}$, is gained by converting a single enput, into a group-invariant enput. In other words, $F_{\mathrm{GP}}$ introduced ambiguity to the dynamical behaviour [43] of $F_{\mathrm{GKL}^{\prime}}$, by allowing the state-transition to optionally occur for a larger set of configurations of a cell's neighbourhood. This redundancy in the form of ambiguity is quite effectively captured by our two-symbol schema redescription with the position-free symbol.

It should be emphasized that until this point, and especially when looking only at the emergent behaviour of these rules, their deep similarity was unknown. With schema redescription of local-level dynamics, not only can we uncover the similarity, but we can explain it. It also makes sense that $F_{\mathrm{GP}}$ was

\footnotetext{
3 redescriptions and dynamics of all the CAs in this paper are available from http: //cnets.indiana.edu/groups/casci/mrecinca/ca
}

obtained via genetic programming [4]. This rule was probably evolved via minor changes to a tree encoding of $F_{\mathrm{GKL}}$; it would need simply to substitute a tree terminal for a sub-tree with a disjunction node for 3 terminal edges, plus a mirror operation to go from $F_{\mathrm{GKL}}$ to $F_{\mathrm{GKL}^{\prime}}$.

We can also see that both rules are extremely canalized: every possible state-change is controlled by two enputs, disregarding the states of all other neighbouring cells. But the $F_{\mathrm{GP}}$ is canalizing in yet another way: it depends, alternatively, on the state of one of the input cells in a group-invariant enput. These group-invariant (collective) enputs can be formally described as a disjunction of alternative input variables (logical literals) and captured in schemata redescription by the additional position-free symbol. This additional form of canalization in Boolean functions, and its effect on determining collective dynamics in automata networks used to model genetic regulation and signalling networks is a topic we will explore further, elsewhere.

Another key idea we will explore in future work is how to use the explanatory power of schema redescription at the local level of Boolean LUTs, to link local to global behaviour in automata networks. From what we presented here, we can already see that the $F_{\mathrm{GP}}$ rule must necessarily lead to some equivalent domains and redundant particle interactions. Since it contains group-invariant enputs, it canalizes distinct and alternative neighbourhood input patterns to the same transition. Therefore, domains that seem to be distinct at the collective space-time behaviour level, can in reality be equivalent and not involved in transmitting novel information through the lattice, merely maintaining the same information in differentlooking domains. In a forthcoming paper we will build up from schema redescription at the local level, and the computation of collective dynamics from incomplete information, to automatically identify domains (relevant and redundant), particles, and particle interactions at the collective dynamics level.

As a final topic of discussion, the present work moves us to consider that an exclusively emergentist approach to complex systems dynamics is incomplete. The induction of recurring patterns in dynamics is not a substitute for uncovering the lower-level mechanisms that actually dictate the dynamics. A full account of complex systems requires attention to local and global dynamics. In a sense, too much attention to the "spots" or "stripes" we observe at the emergent level of behaviour, can mask that underneath it all there may be a very similar animal.

\section{ACKNOWLEDGMENTS}

This work was supported by Fundação para a Ciência e a Tecnologia (Portugal) grant 36312/2007. We thank the FLAD Computational Biology Collaboratorium at the Gulbenkian Institute (Portugal) for hosting and providing facilities used for this research. We also thank Indiana University for providing access to its computing facilities. 


\section{REFERENCES}

[1] M. Mitchell, J. Crutchfield, and R. Das, "Evolving cellular automata with genetic algorithms: A review of recent work," in Proceedings of the First International Conference on Evolutionary Computation and its Applications (EvCA'96). Russian Academy of Sciences, 1996.

[2] P. Gacs, L. Kurdyumov, and L. Levin, "One-dimensional uniform arrays that wash out finite islands," Probl. Peredachi. Inform., vol. 14, pp. 92 98, 1978.

[3] P. Gonzaga de Sá and C. Maes, "Gacs-kurdyumov-levin automaton revisited," Journal of Statistical Physics, vol. 67, no. 3-4, pp. 507-522, 1992.

[4] D. Andre, F. B. III, and J. Koza, "Discovery by genetic programming of a cellular automata rule that is better than any known rule for the majority classification problem," in Proceedings of the First Annual Conference on Genetic Programming, J. Koza, D. Goldberg, and D. Fogel, Eds. MIT Press, 1996, pp. 3-11.

[5] S. J. Heims, The Cybernetics Group. MIT Press, 1991.

[6] W. McCulloch and W. Pitts, "A logical calculus of ideas immanent in nervous activity," Bulletin of Mathematical Biophysics, vol. 5, pp. 115133, 1943.

[7] S. Kauffman, "Metabolic stability end epigenesis in randomly constructed genetic nets." Journal of Theoretical Biology, vol. 22, pp. 437$467,1969$.

[8] B. Derrida and D. Stauffer, "Phase transitions in two-dimensional kauffmann cellular automata," Europhys. Lett., vol. 2, pp. 739-745, 1986.

[9] S. Kauffmann, The Origins of Order: Self-Organization and Selection in Evolution. Oxford University Press, 1993.

[10] L. Mendoza and E. R. Alvarez-Buylla, "Dynamics of the genetic regulatory network for arabidopsis thaliana flower morphogenesis." $J$ Theor Biol, vol. 193, no. 2, pp. 307-319, Jul 1998. [Online]. Available: http://dx.doi.org/10.1006/jtbi.1998.0701

[11] R. Albert and H. Othmer, "The topology of the regulatory interactions predicts the expression pattern of the segment polarity genes in drosophila melanogaster." J Theor Biol, vol. 223, no. 1, pp. 1-18, Jul 2003.

[12] C. Espinosa-Soto, P. Padilla-Longoria, and E. R. Alvarez-Buylla, "A gene regulatory network model for cell-fate determination during arabidopsis thaliana flower development that is robust and recovers experimental gene expression profiles." Plant Cell, vol. 16, no. 11, pp. 2923-2939, Nov 2004. [Online]. Available: http://dx.doi.org/10.1105/tpc.104.021725

[13] S. Kauffman, C. Peterson, B. Samuelsson, and C. Troein, "Random boolean network models and the yeast transcriptional network." Proce, vol. 100, no. 25, pp. 14796-14799, Dec 2003. [Online]. Available: http://dx.doi.org/10.1073/pnas.2036429100

[14] M. Chaves, R. Albert, and E. D. Sontag, "Robustness and fragility of boolean models for genetic regulatory networks." J Theor Biol, vol. 235, no. 3, pp. 431-449, Aug 2005. [Online]. Available; http://dx.doi.org/10.1016/j.jtbi.2005.01.023

[15] S. Bornholdt, "Systems biology: less is more in modeling large genetic networks," Science, Jan 2005. [Online]. Available: http: //stke.sciencemag.org/cgi/content/summary/sci;310/5747/449

[16] - "Boolean network models of cellular regulation: prospects and limitations," J R Soc Interface, vol. 5 Suppl 1, pp. S85-94, Aug 2008.

[17] A.-L. Barabási, Linked: The New Science of Networks. New York, USA: Perseus Books, 2002

[18] M. Newman, A.-L. Barabási, and D. J. Watts, The Structure and Dynamics of Networks. Princeton, NJ: Princeton University Press, 2006.

[19] U. Alon, "Network motifs: theory and experimental approaches." Nat Rev Genet, vol. 8, no. 6, pp. 450-461, Jun 2007. [Online]. Available: http://dx.doi.org/10.1038/nrg2102

[20] M. Mitchell, "Complex systems: Network thinking," Artificial Intelligence, vol. 170, no. 18, pp. 1194-1212, 2006.

[21] D. Peak, J. D. West, S. M. Messinger, and K. A. Mott, "Evidence for complex, collective dynamics and distributed emergent computation in plants," Proceedings of the National Academy of Sciences, vol. 101, no. 4, pp. 918-922, 2004

[22] T. Helikar, J. Konvalina, J. Heidel, and J. A. Rogers, "Emergent decision-making in biological signal transduction networks." Proc Natl Acad Sci U S A, vol. 105, no. 6, pp. 1913-1918, Feb 2008. [Online]. Available: http://dx.doi.org/10.1073/pnas.0705088105
[23] M. Marques-Pita, M. Mitchell, and L. M. Rocha, "The role of conceptual structure in designing cellular automata to perform collective computation," in Unconventional Computation. 7th International Conference. LNCS 5204, C. Calude, J. F. Costa, R. Freund, M. Oswald, and G. Rozenberg, Eds., vol. 5204, 2008, pp. 146-163.

[24] M. Marques-Pita and L. M. Rocha, "Conceptual structure in cellular automata: The density classification task," in Proceedings of the Eleventh International Conference on Artificial Life (Alife XI), S. Bullock, J. Noble, R. A. Watson, and M. A. Bedau, Eds. MIT Press, Cambridge, MA., 2008, pp. 390-397.

[25] C. J. O. Reichhardt and K. Bassler, "Canalization and symmetry in boolean models for genetic regulatory networks," Physica A, vol. 40, pp. 4339-4350, 2007.

[26] J. von Neumann, Theory of Self-Reproducing Automata, ser. Edited and completed by A. W. Burks. University of Illinois Press, 1966.

[27] J. Grefenstette, S. Kim, and S. Kauffman, "An analysis of the class of gene regulatory functions implied by a biochemical model," BIOSYSTEMS, Jan 2006. [Online]. Available: http://linkinghub.elsevier. com/retrieve/pii/S0303264705001681

[28] J. Holland, K. Holyoak, R. Nisbett, and P. Thagard, Induction: Processes of Inference, Learning and Discovery. MIT Press, 1986.

[29] W. V. Quine, "A way to simplify truth functions," The American Mathematical Monthly, vol. 62, no. 9, pp. 627-631, 1955.

[30] L. Valiant, "A theory of the learnable," Communications of the ACM, Jan 1984. [Online]. Available: http://portal.acm.org/citation.cfm?id= 1968.1972

[31] E. McCluskey, "Detection of group invariance or total symmetry of a boolean function," Bell System Technical Journal, vol. 35, no. 6, pp. $1445-1453,1956$.

[32] M. Mitchell, J. Crutchfield, and P. Hraber, "Revisiting the edge of chaos: Evolving cellular automata to perform computations," Complex Systems, vol. 7, pp. 89-130, 1993.

[33] J. Hanson, "Computational mechanics of cellular automata," Ph.D. dissertation, University of California, Berkeley, 1993.

[34] J. Crutchfield, "Is anything ever new? considering emergence," in Complexity: Metaphors, Models, and Reality,, ser. SFI Series in the Sciences of Complexity, G. Cowan and D. Pines, Eds. Addison-Wesley, 1994, vol. XIX, pp. 479-497.

[35] J. P. Crutchfield, M. Mitchell, and R. Das, "The evolutionary design of collective computation in cellular automata," in Evolutionary Dynamics-Exploring the Interplay of Selection, Neutrality, Accident, and Function, J. P. Crutchfield and P. K. Schuster, Eds. Oxford University Press, New York, 2003, pp. 361-411.

[36] L. M. Rocha and W. Hordijk, "Material representations: From the genetic code to the evolution of cellular automata," Artificial Life, vol. 11, no. 1-2, pp. $189-214$, Winter/Spring 2005.

[37] J. T. Lizier, M. Prokopenko, and A. Y. Zomaya, "Local information transfer as a spatiotemporal filter for complex systems," Physical Review $E$, vol. 77, p. 026110, 2008.

[38] C. Shalizi, R. Haslinger, J.-B. Rouquier, K. Klinkner, and C. Moore, "Automatic filters for the detection of coherent structure in spatiotemporal systems," Physical Review E, vol. 73, 2006.

[39] M. Marques-Pita, R. Manurung, and H. Pain, "Conceptual representations: What do they have to say about the density classification task by cellular automata?" in ECCS'06, European Conference on Complex Systems., J. Jost, F. Reed-Tsotchas, and P. Schuster, Eds., 2006.

[40] M. Mitchell, J. Crutchfield, and P. Hraber, "Evolving cellular automata to perform computations:mechanisms and impediments," Physica D, vol. 75, pp. 361-391, 1994.

[41] W. Hordijk, J. P. Crutchfield, and M. Mitchell, "Mechanisms of emergent computation in cellular automata," in Proceedings of the Fifth International Conference on Parallel Problem Solving From Nature-PPSN V, A. E. Eiben, Ed. New York: Springer, 1998.

[42] D. Wolz and P. B. de Oliveira, "Very effective evolutionary techniques for searching cellular automata rule spaces," Journal of Cellular Automata, vol. 3, pp. 289-312, 2008.

[43] G. Klir and M. Wierman, Uncertainty-Based Information : Elements of Generalized Information Theory, ser. Studies in Fuzziness and Soft Computing. Physica-Verlag, 1999. 